Brit. J. industr. Med., 1959, 16, 318.

\title{
LUNG CANCER IN COAL-MINERS
}

\author{
BY \\ R. ABBEY SMITH \\ From the Thoracic Surgical Unit, King Edward VII Memorial Chest Hospital, Warwick
}

(RECEIVED FOR PUBLICATION NOVEMBER 5, 1958)

In a study of coal-miners suffering from lung cancer two features of special interest are recorded. The difficulties in diagnosis are illustrated by case reports. The two-year survival rate after surgical removal of the tumour is significantly better in coal-miners than in non-miners. After operation $87 \%$ of coal-miners were alive two years later, compared with only $36 \%$ of other patients operated upon. It is suggested that the rate of spread of the tumour to the mediastinal structures, lymph glands, and blood vessels is retarded by the effects of pre-existing dust disease in the lungs. This factor explains the good results in a group of patients selected for surgery.

Surprisingly little information is available on the clinical aspects of the association of lung cancer and coal-miners' dust disease. From a study of 78 coalminers with lung cancer, a good proportion of whom have been operated on, two points for discussion arise: one, the difficulty in reaching the correct diagnosis at a stage when surgical removal of the lung tumour is possible; the other, the high proportion of coal-miners surviving two or more years after resection compared with the proportion of survivors in non-miners. The figures under consideration are small, but this trend seems definite and because survival after operation has, so far as can be ascertained, never been related to this occupation, the results are discussed from the point of view of factors involved in the control of lung cancer by operation.

Since we are associating post-operative survival solely with the occupation of coal-mining, the difficult correlation of the degree of occupational pneumoconiosis with length of survival after operation will not be attempted; nor is it desirable to speculate on results in welders, smiths, foundryworkers, and stonemasons, workers in whom survival has been longer than anticipated. We shall define a coal-miner simply as a person who has worked five years or more underground at the coalface, not necessarily in the Warwickshire or south Derbyshire mines, where most of the patients were occupied. Possible differences in the properties of dust from different geographical regions have not been considered.

\section{Diagnosis of Lung Cancer in Miners}

In a paper on "Silicotic and Tuberculo-silicotic Lesions Simulating Bronchogenic Carcinoma",
Kergin (1952) described eight patients with unilateral radiological densities of the type usually seen in lung carcinoma which were, in fact, caused by silicosis or tuberculo-silicosis. He referred to eariier papers by Woodruff and Kelley (1947) and Bradshaw and Chodoff (1939). James (1955) mentioned the difficulty in diagnosing lung cancer in the presence of massive pneumoconiosis and Kilpatrick, Heppleston, and Fletcher (1954), discussing the differential diagnosis of cavitated coalworkers' progressive massive fibrosis, state that a cavitated bronchial carcinoma is probably the most difficult to exclude. When routine methods fail to confirm a diagnosis the cause of difficulty, we find, is either the slowness in growth of certain lung cancers in miners, or the occasional occurrence of a single radiological shadow due to a localized massive fibrotic lesion without any evidence of simple pneumoconiosis. That other radiological evidence of pneumoconiosis may be absent is important and perhaps this feature is not sufficiently widely recognized. Two examples illustrating this will be described, closely resembling the case reported recently by Davis and Snow (1958). These authors believe that a new classification of pneumoconiosis is required to include solitary lesions occurring in lungs which otherwise show only equivocal findings of pneumoconiosis. The facts support the introduction of this amendment. It has also been observed that the course of some of the diseases, commonly confused with carcinoma, may be modified in miners. Familiarity with these points only increases awareness that mistakes may be made without clarifying any particular problem. In diagnosis, observation of the patient's radiological changes over a period is not helpful. So slowly can the tumour progress that one patient 
with a proved carcinoma continued at work for seven years after diagnosis. More active steps are required, provided the clinician accepts that even at thoracotomy differentiation between progressive massive fibrosis, tuberculosis, and carcinoma may not be possible. Kergin (1952) has written about this in detail. Case histories of five patients where doubt existed will illustrate these points. Cases 1 and 2 are examples of localized massive fibrosis thought to be tumour. Cases 3 and 4 demonstrate the insidious advance of the tumour when associated with pneumoconiosis, and Case 5 the manner in which pneumoconiosis may obscure the characteristics of another disease.

\section{Case Reports}

Case 1.-T.W., aged 60, was a coal-face worker in a Durham hard coal-mine for 20 years. He retired permanently from mining in 1930 and had never had a chest radiograph taken before 1952. His chest was radiographed then because of general malaise. The film is shown (Fig. 1). A diagnosis of carcinoma was made and the right upper lobe removed. Post-operative examination of the lobe showed a cavity $2.5 \mathrm{~cm}$. in diameter filled with jet black, thin fluid. Some collections of dust with little reaction were present, with denser fibrotic areas around the cavity. There was no evidence of carcinoma. The patient has remained well and his chest radiograph six years after operation is shown (Fig. 2). It may be noted that his last exposure to coal dust was 22 years before operation.

Case 2.-W.R., aged 72, had worked for 50 years in the Warwickshire coal-mines. He was referred in September, 1955, because of a shadow in the chest radiograph, which was detected when the patient reported

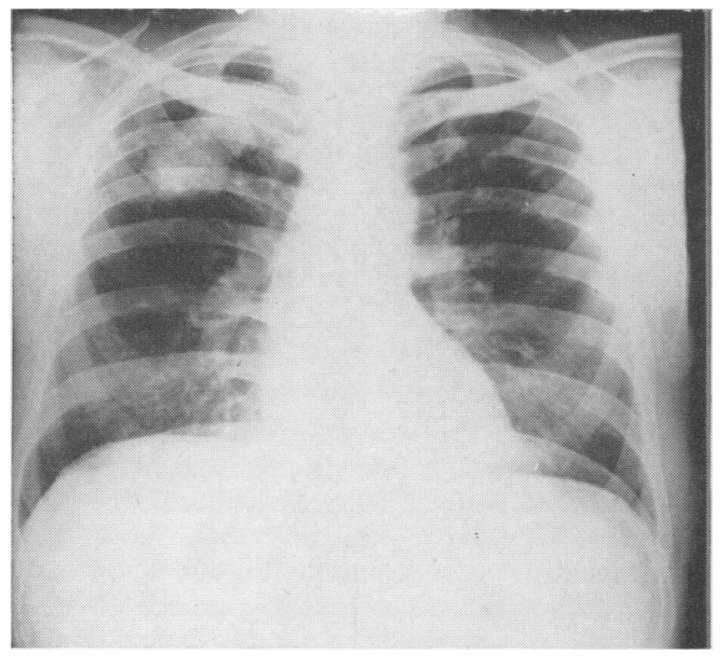

Fig. 1.-Case 1. Mass in right upper lobe thought to be carcinoma. No evidence of pneumoconiosis. with a history of productive cough. There had been no haemoptysis. The radiological lesion increased and the film in November, 1955, is shown (Fig. 3.) At about this time the patient had a number of small haemoptyses and although bronchoscopy showed no abnormality, a diagnosis of lung carcinoma was made. In view of the patient's age and absence of symptoms, operation was not advised. The shadow increased slowly in size until February, 1956, when the patient had a further haemoptysis while at work and coughed up a large amount of thin, black sputum. The shadow resolved and the patient is now in his 53rd year of mining. His chest radiograph in 1958 is shown (Fig. 4).

Case 3.-R.C., aged 44 years, worked as a Warwickshire coal-miner for 25 years. His first radiograph in 1955 during a routine radiological investigation showed a small nodule in the left mid-zone on a background of simple pneumoconiosis (Fig. 5). During the next three years the nodule increased in size, although the patient was at work and symptomless. He was seen in 1958 with the picture shown in Fig. 6. There was no confirmatory evidence of tumour, but the lesion was regarded as such and the left upper lobe removed, three years after the date of the original radiological abnormality. The pathological report on the left upper lobe reads . . .

"In the posterior segment there is a firm white growth $3 \mathrm{~cm}$. in diameter arising in relation to the segmental bronchus. The remainder of the lobe contains scattered firm pale nodules up to $2 \mathrm{~mm}$. in diameter, but there is no particular concentration of nodules or of more massive fibrosis in the immediate vicinity of the growth. Numerous small anthracotic areas are also present throughout the lobe. The histological appearances of this specimen are complex. The growth is a bronchiolar cell carcinoma and the firm pale nodules are also tumour foci of similar structure to the main growth. There is no indication as to whether they are foci of multicentric

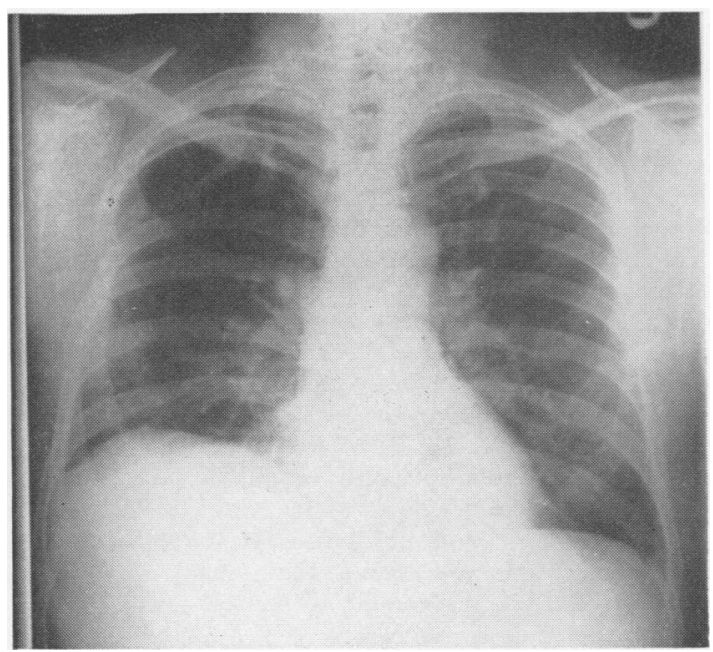

Fig. 2.-Case 1. Six years after resection of right upper lobe. 


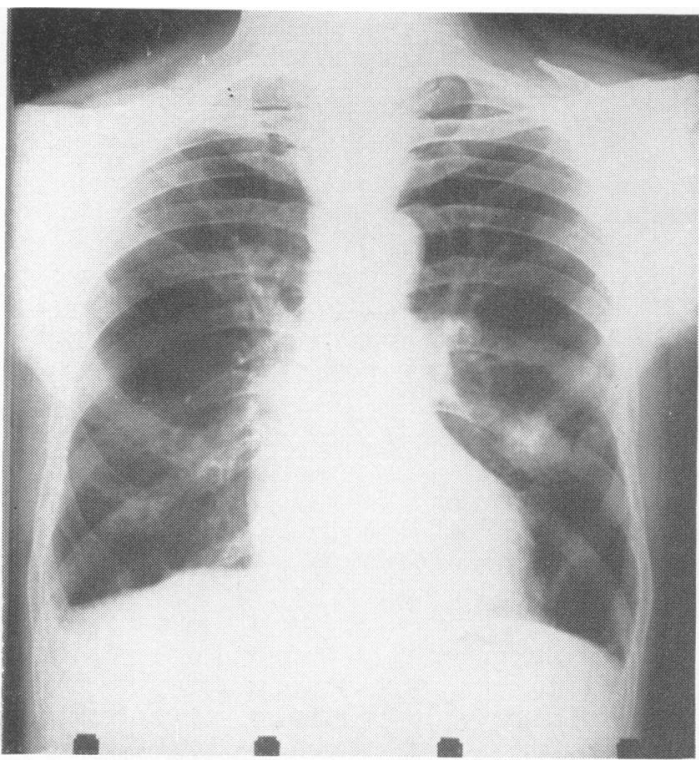

FIG. 3.-Case 2. Mass in left lower lobe thought to be carcinoma. No evidence of pneumoconiosis.

tumour or metastases. The small anthracotic areas are foci of simple pneumoconiosis. There are many scattered areas of pneumonitis affecting primary lobules. All combinations of these three lesions are seen in close juxtaposition in different parts of the lobe, but the relationships appear to be accidental. Lymph nodes show anthracosis and metastatic tumour."

The specimen is shown in Fig. 7.

Convalescence from the operation was uneventful and the patient has returned to his previous occupation.

Case 4.-W.J., aged 64, was employed in a South Wales hard coal-mine from 1911 to 1938 . He retired in 1938. His first radiograph was in 1947. This film showed simple pneumoconiosis and a disability pension was granted. In 1955 more solid-looking shadows appeared in the right lower zone (Fig. 8). During the next 10 weeks these shadows fluctuated in intensity but never cleared. Bronchoscopy showed a normal bronchial tree. A diagnosis of carcinoma of the right lower lobe was made and operation advised. At operation the whole lung was infiltrated with small hard nodules, with a more confiu nnt mass in the basal segments of the lower lobe. An accurate distinction between carcinoma, tuberculosis, or progressive massive fibrosis, or any combination of these diseases, was not possible. The right lower lobe was removed. Pathological examination showed the lesion to be an epidermoid carcinoma. The hilar glands were anthracotic and fibrosed. At the present time, three years since operation, the patient works as a gardener and has no significant increase in dyspnoea.

Case 5.-H.H., aged 46, a Warwickshire miner for 23 years, had miniature chest films in 1947 and 1954 which were reported as normal. In 1958 he was found to have

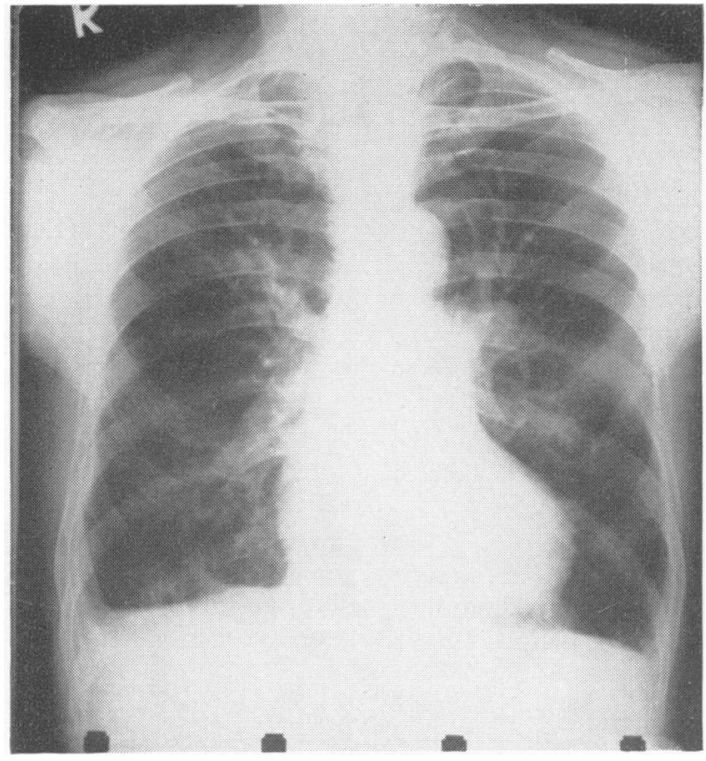

FIG. 4.-Case 2. Present state. Complete resolution of left lower lobe shadow.

a circumscribed, rounded shadow in the left lower lobe. The patient was symptom free and a full investigation was not contributory. There seemed no doubt that the lesion was intrapulmonary and as the chest film in 1954 was normal (it was re-perused by Dr. Gordon Evans), a provisional diagnosis of carcinoma was made. At operation the left lung was extensively infiltrated by small hard nodular areas of pneumoconiosis. In the lower lobe a mass about $2.5 \mathrm{~cm}$. in diameter of the same consistency was present. Its characteristics were obscured by the surrounding nodules. A diagnosis by palpation was not possible. The mass was incised; it was thought to be fixed within the lung and a diagnosis of carcinoma was made. A frozen section was considered unnecessary. The lower lobe was removed. Pathological examination revealed the tumour to be a chondromatous hamartoma. In an otherwise normal lung the mobility of this tumour is pathognomonic. Its fixity and loss of definition were due to the pre-existing pneumoconiosis. No ready explanation for the normal appearances in the 1954 chest film is available.

\section{The High Survival Rate in Coal-miners}

In the post-operative follow-up of all patients with lung cancer operated on in this hospital, it appeared that patients from agricultural and rural areas generally fared less well than those from industrial districts. A reason for this was sought and it became apparent that the group from the industrial districts included a number of coal-miners. Comparison of the figures for all patients operated on with those for coal-miners is of interest. Of a 


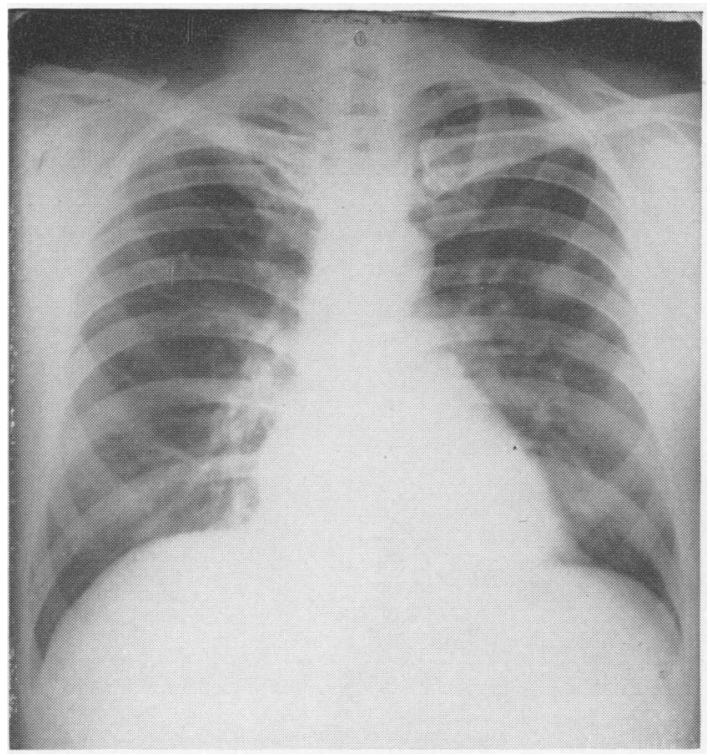

Fis. 5
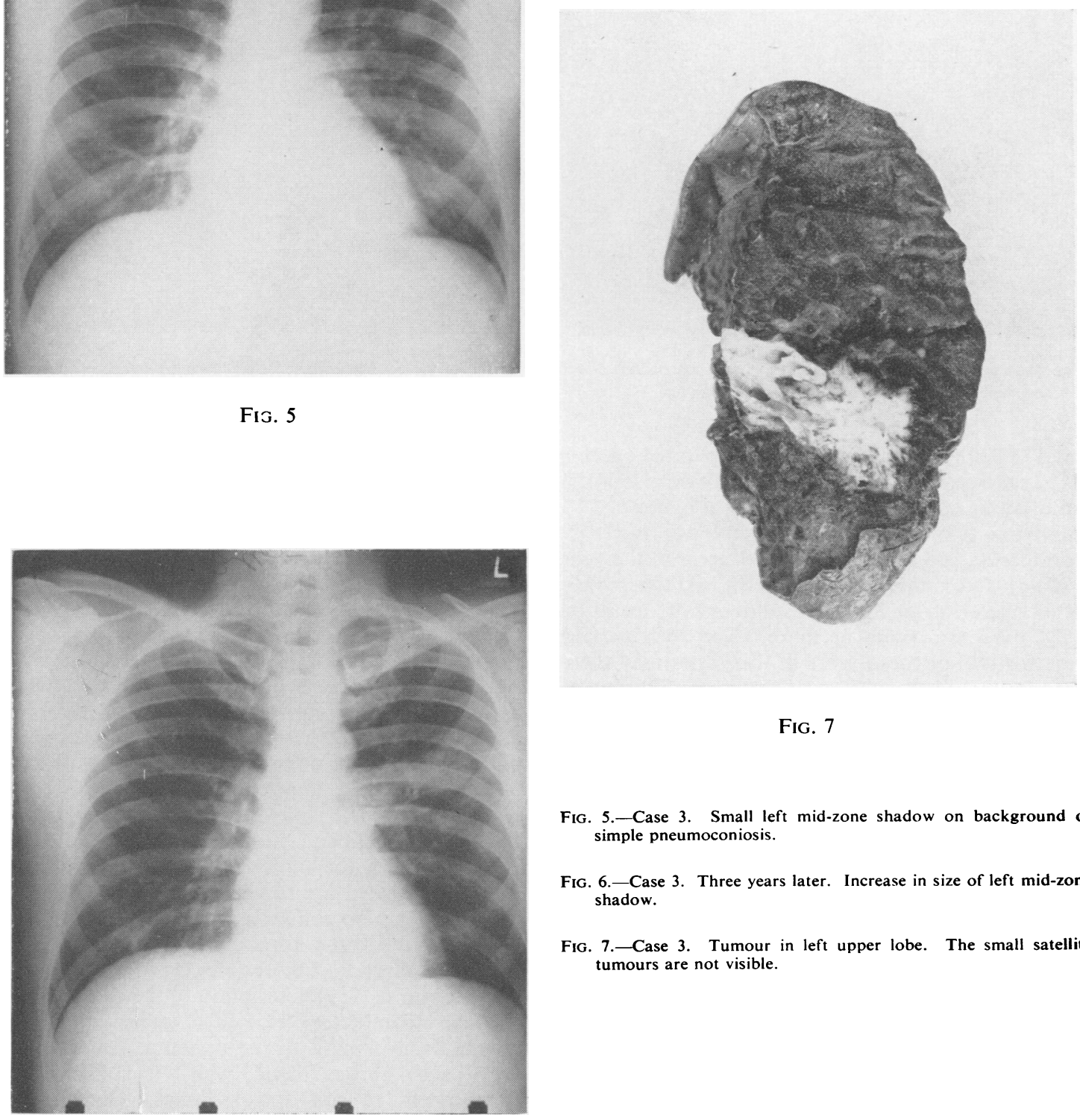

FIG. 7

FIG. 5.-Case 3. Small left mid-zone shadow on background of simple pneumoconiosis.

FIG. 6.-Case 3. Three years later. Increase in size of left mid-zone shadow.

Fig. 7.-Case 3. Tumour in left upper lobe. The small satellite tumours are not visible.

FIG. 6 


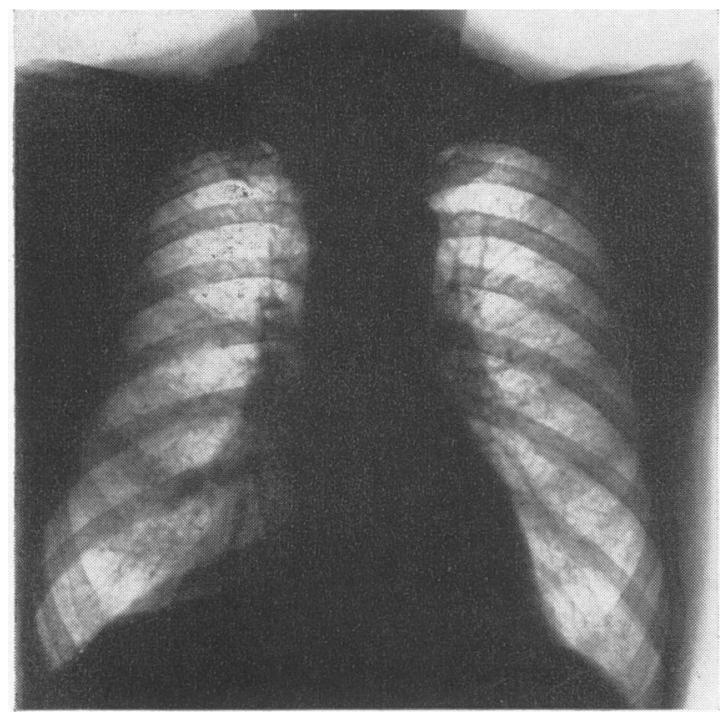

Fig. 8.-Case 4. Simple pneumoconiosis with increased shadow in the right lower lobe due to carcinoma of the lung.

total of 320 patients from all areas operated on, only $36 \%$ survived two years or more. Taylor (1954), in an analysis of 512 resections, gives a figure of $34 \%$ surviving two years. Bignall and Moon (1955), considering 205 patients operated upon in the years $1950-51$, found that $10 \%$ died in the first two months after operation and of the survivors of operation, $47 \%$ lived two years or more. The $36 \%$ survival rate for two or more years in this series may therefore be regarded as an average figure. During the same period with the same criteria of operability, 21 miners have undergone resection and of these only three have died in the first two years; this represents an $86 \%$ two-year survival rate, and indicates that in our experience a coal-miner has an appreciably greater chance of survival after operation than the non-miner. To assume that the results of surgery for lung cancer in predominantly coalmining areas should therefore be correspondingly better than from other areas may be fallacious. The figure may be loaded against better results in the mining areas by increased deaths in the first two years from respiratory insufficiency, or from factors in selection of cases. If, however, the findings in this group of miners are significant, it implies that deaths directly attributable to extension of the tumour should be below average in mining areas. No published figures are available to substantiate this hypothesis.

The results in this study are not to be explained by selection of only the most favourable cases for

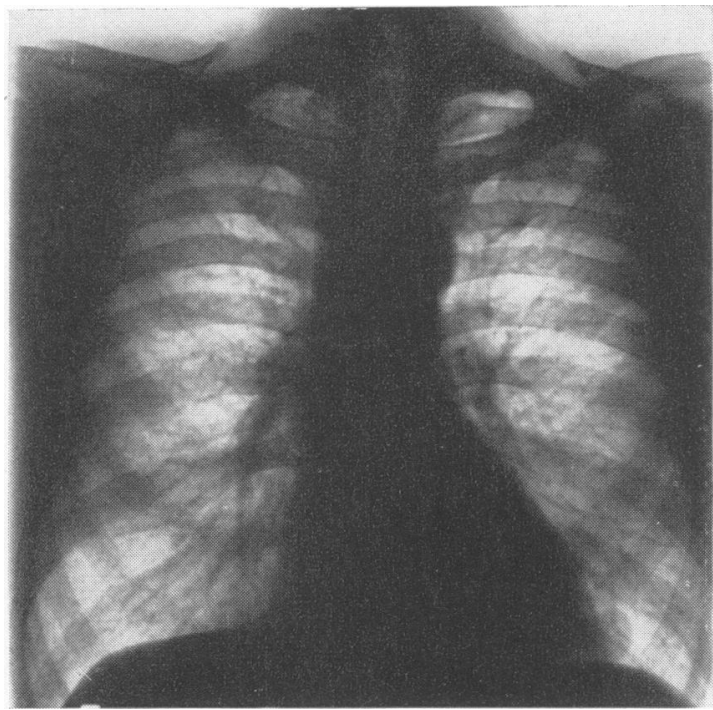

Fig. 9.-Case 5. Circumscribed left lower lobe shadow at time of operation. Thought to be lung carcinoma. Chest radiograph in 1954 known to be normal.

operation out of all the miners referred for surgery. The two most likely explanations of the results in miners are that the rate of local growth and spread of the tumour are considerably retarded by the effects of prolonged inhalation of coal dust on the lung parenchyma and lymphatics, or that the coalminer develops a specific and slow-growing type of tumour. James (1955) found no evidence to support the second explanation from microscopic examination of a large number of tumours from coal-miners at necropsy. The former explanation is the more attractive. It may be related to the ill-understood phenomenon that a history of some six months before diagnosis improves the prognosis after resection (Bignall and Moon 1955), or without treatment (Buchberg, Lubliner, and Rubin 1951), compared with the tumour of short history. The numbers resected are too small for any worthwhile analysis of pre-operative length of history, glandular involvement at the time of operation, or other statistical division, but the operative findings confirm Kergin's (1952) description of the characteristic chronic inflammatory type of reaction with lymph glands firmly bound to the vessels and bronchi by very dense fibrous tissue, which is so frequently encountered in coal-miners' lungs. Kergin also draws attention to the fibrosis around the bronchovascular bundles, the sclerosis of vessels, and the obliteration of lymphatic channels. These gross and microscopic characteristics of the miner's lung offer a possible reason for the retardation in the 
local growth and spread of the tumour from the time of its origin. The unfavourable medium which this diffuse fibrosis represents may well depress the vitality of the tumour. Comparison with the changes produced in the lung and its vessels and lymphatics by radiation therapy is a subject for further study. The favourable post-operative result in coal-miners merits further consideration.

I am greatly indebted to Mr. H. V. Wingfield, who operated on Case 1, and to Dr. A. P. Prior and Dr. M. K. Alexander for the pathological reports.

The patients were referred by Dr. E. Calvey, Dr. Gordon Evans, and Dr. J. Sharkey.
I am grateful to Miss D. Beauchamp for her help in collecting information about the patients' follow-up.

\section{REFERENCES}

Bignall, J. R., and Moon, A. J. (1955). Thorax, 10, 183.

Bradshaw, H. H., and Chodoff, R. J. (1939). Amer. Rev. Tuberc., 39, 817 .

Buchberg, A., Lubliner, R., and Rubin, E. H. (1951). Dis. Chest, 20,257 .

Davis, W. C., and Snow, W. T. (1958). J. thorac. Surg., 36, 185.

James, W. R. L. (1955).' Brit. J. industr. Med. 12, 87.

Kergin, F. G. (1952). J. thorac. Surg., 24, 545.

Kilpatrick, G. S., Heppleston, A. G., and Fletcher, C. M. (1954). Thorax, 9, 260. Taylor, A. Brian'(1954). Annual Cancer Report, United Birmingham

Woodruff, W., and Kelley, W. O. (1947). J. thorac. Surg., 16, 282. 